\title{
Food Webs, Competition Graphs, and Habitat Formation
}

\author{
M. Cozzens * \\ DIMACS, Rutgers University, 96 Frelinghuysen Road, Piscataway, NY 08854-8018, USA
}

\begin{abstract}
One interesting example of a discrete mathematical model used in biology is a food web. The first biology courses in high school and in college present the fundamental nature of a food web, one that is understandable by students at all levels. But food webs as part of a larger system are often not addressed. This paper presents materials that can be used in undergraduate classes in biology (and mathematics) and provides students with the opportunity to explore mathematical models of predator-prey relationships, determine trophic levels, dominant species, stability of the ecosystem, competition graphs, interval graphs, and even confront problems that would appear to have logical answers that are as yet unsolved.
\end{abstract}

Key words: food web, predators and prey, dominance, trophic level, trophic status, mathematical model, graph, directed graph, competition graph, interval graph, boxicity

AMS subject classification:

\section{Goals:}

- Recognize various relationships between organisms, and look for patterns in food webs.

- Use graphs and directed graphs to model complex trophic relationships.

- Determine trophic levels and status within a food web, and the significance of these levels in calculating the relative importance of each species (vertices) and each relationship (arcs) in a food web.

- Use the food web and corresponding competition graph to determine the dimension of the community's habitat.

${ }^{*}$ Corresponding author. E-mail: midgec@ dimacs.rutgers.edu 


\section{Introduction}

Early on, the relationships between mathematics and the physical sciences have been appreciated and often have been used as a reason to study mathematics and its applications to the physical sciences. However, the interplay between mathematics and the biological sciences was understood by only a few. The uses of mathematical models in the biological sciences were restricted to continuous models, most often using a dynamical systems approach. All this has changed! Increasingly, many biological phenomena are being viewed as involving the processing of information, which ultimately involves using discrete mathematics. Technology has made working with large data sets possible, thus modern mathematical and information sciences have played an important role in many major biological accomplishments. For example no place is this more true than in sequencing the human genome, and making mathematical models of fundamental importance in the rapidly-expanding and evolving concept of "digital biology" [3]. In discrete models, since data is discrete, this data does not have to be smoothed out, or points eliminated, to allow for mathematical models to be applied.

One example of a discrete mathematical model used in biology is that of a food web. The first biology courses in high school and in college present the fundamental nature of a food web, one that is understandable by students at all level. But food webs as part of a larger system are often not addressed. This paper presents materials that can be used in undergraduate classes in biology (and mathematics) and provides students with the opportunity to explore mathematical models of predator-prey relationships, determine trophic levels, dominant species, stability of the ecosystem, and even confront problems that would appear to have logical answers that are as yet unsolved.

\section{Modeling Predator-Prey Relationships with Food Webs}

\subsection{Food Webs}

Have you ever played the game Jenga? It's a game where towers are built from interwoven wooden blocks, and each player tries to remove a single block without the tower falling. The player who crashes the tower of blocks loses the game.

Food webs are towers of organisms. Each organism depends for food on one or many other organisms in an ecosystem. The exceptions are primary producers - the organisms at the foundation of the ecosystems that produce their energy from sunlight through photosynthesis or from chemicals through chemosynthesis. Factors that limit the success of primary producers are generally sunlight, water, or nutrient availability. These are physical factors that control a food web from the "bottom up." On the other hand, certain biological factors can also control a food web from the "top down." For example, certain predators, such as a shark, lion, wolf, or a human can suppress or enhance the abundance of other organisms. They can suppress them directly by eating their prey or indirectly by eating something that would eat something else. Understanding the difference between direct and indirect interactions within ecosystems is critical to building food webs. For example, suppose your favorite food is a hamburger. The meat came from a cow, but 
a cow is not a primary producer-it can't photosynthesize! But a cow eats grass, and grass is a primary producer. So, you eat cows, which eat grass. This is a simple food web with three players. If you were to remove the grass, you wouldn't have a cow to eat. So, the availability and growth of grass indirectly influences whether or not you can eat a hamburger. On the other hand, if cows were removed from the food web, then the direct link to your hamburger would be gone, even if grass persisted.

Primary producers are always at the bottom of the food web. Above the primary producers are various types of organisms that exclusively eat plants. These are considered to be herbivores, or grazers. Animals that eat herbivores, or each other, are carnivores, or predators. Animals that eat both plants and other animals are omnivores. An animal at the very top of the food web is called a top predator.

Through the various interactions in a food web, energy gets transferred from one organism to another. Food webs, through both direct and indirect interactions, describe the flow of energy through an ecosystem. By tracking the energy flow, you can derive where the energy from your last meal came from, and how many species contributed to your meal. Understanding food webs can also help to predict how important any given species is, and how ecosystems change with the addition of a new species or removal of a current species. Food webs are complex! In this module, you will explore the complexity of food webs in mathematical terms, using a physical model, called a directed graph (digraph), to map the interactions between organisms.

A digraph represents the species in an ecosystem as points or vertices (singular $=$ vertex) and puts arrows for arcs from some vertices to others depending on the energy transfer, from a prey to a predator of that prey.

The species that occupy an area and interact either directly or indirectly form a community. The mixture and characteristics of these species define the biological structure of the community. These include parameters such as feeding patterns, abundance, population density, dominance, and diversity. Acquisition of food is a fundamental process of nature providing both energy and nutrients. The interactions of species as they attempt to acquire food determine much of the structure of a community. We use food webs to represent these feeding relationships within a community.

Remark 1. Example 1 In the partial food web below, sharks eat sea otters, sea otters eat sea urchins and large crabs, large crabs eat small fishes, and sea urchins and small fishes eat kelp. Said in another way, sea urchins and large crabs are eaten by sea otters (both are prey for sea otters) and sea otters are prey for sharks. These relationships are modeled by the food web shown in Figure 1.

Activity 1. Create a food web from the following predator-prey table (Table 1 is related to the food web shown in Figure 1). There are various tools online that are used to construct food webs from data of this nature, but this time, try it by hand. 


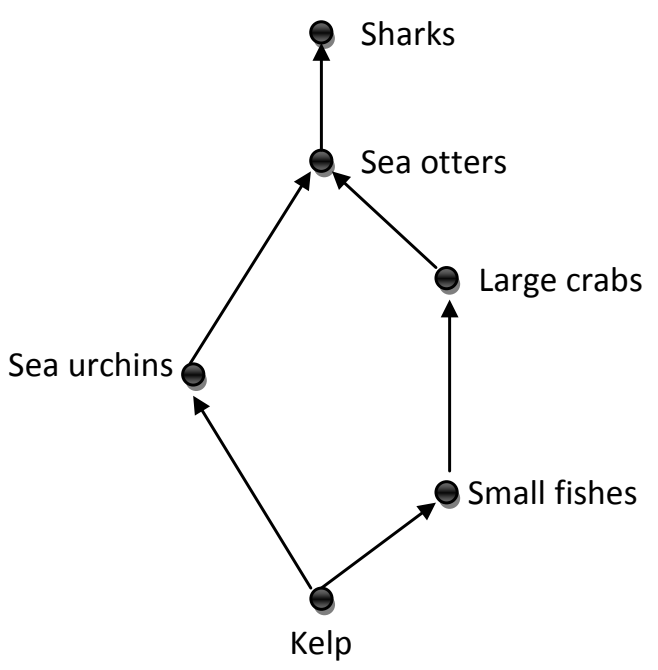

Figure 1: Food web.

Remark 2. Question Are there any species that are only predators and not prey, any that are prey that are not predators? What are they?

\subsection{Trophic Levels and Trophic Status}

Now let's consider where a species is located "in the food chain" or specifically in the food web. For example, producers, such as kelp in our first example in Figure 1, are at the bottom of the food chain; sharks appeared at the top. If it is a food chain, where one and only one species is above the other, it is easy, but what if the food web is more complex as in our first example and represents a whole community?

Trophic levels in food webs provide a way of organizing species in a community food web into feeding groups. Scientists have used various methods in classifying species in a food web into these various feeding groups. The most elementary way is to divide them into the following categories: primary producers, secondary producers and consumers, the latter which are then divided into herbivores and carnivores based on their consumption of plant or animal products. This makes the assumption that there are at most three (trophic) levels in a food web. It also opens the question of how to classify consumers such as bears who are omnivores. Most books group omnivores with carnivores, but is there a scientific basis for doing so? Other scientists consider the positioning in the food web to be most important: a consumer is at a higher level than what it consumes. They then define trophic levels as follows: a species that is never a predator is at trophic level 0, one who preys on that species is at level 1, etc. Determining trophic level in a food chain is easy under this latter definition. For example, for the food chain below, kelp is at trophic level 0, sea urchins are at trophic level 1, sea otters are at trophic level 2, and sharks are at trophic level 3.

Food webs, however, are not necessarily food chains like in Figure 2, but they are a mixture of multiple food chains meshed together. Determining trophic level for more complex food webs 


\begin{tabular}{|c|c|}
\hline Species & Species they feed on \\
\hline Shark & Sea otter \\
\hline Sea otter & $\begin{array}{l}\text { Sea stars, sea urchins, large crabs, large fish and } \\
\text { octopus, abalone }\end{array}$ \\
\hline Sea stars & Abalone, small herbivorous fishes, sea urchins \\
\hline Sea urchins & $\begin{array}{l}\text { Kelp, sessile invertebrates, drift algae and dead ani- } \\
\text { mals }\end{array}$ \\
\hline Abalone & Drift algae and dead animals \\
\hline Large crabs & $\begin{array}{l}\text { Sea stars, smaller predatory fishes and invertebrates, } \\
\text { drift algae and dead animals, small herbivorous fishes } \\
\text { and invertebrates, kelp }\end{array}$ \\
\hline Smaller predatory fishes & Sessile invertebrates, planktonic invertebrates \\
\hline $\begin{array}{l}\text { Small herbivorous fishes and } \\
\text { invertebrates }\end{array}$ & Kelp \\
\hline Kelp & - \\
\hline Large fish and octopus & Smaller predatory fishes and invertebrates \\
\hline Sessile invertebrates & $\begin{array}{l}\text { Microscopic planktonic algae, planktonic inverte- } \\
\text { brates }\end{array}$ \\
\hline algae and dead animals & Kelp, sessile invertebrates \\
\hline Planktonic invertebrates & Microscopic planktonic algae \\
\hline Microscopic planktonic algae & - \\
\hline
\end{tabular}

Table 1: Sea otter food web data.

(ones that are not food chains) is more difficult. In fact, the number of trophic levels in a food web is sometimes used as a measure of complexity, as we will see in the next section. But since food webs can be represented as digraphs, we can use some properties of these digraphs to determine trophic level in any kind of food web. Fortunately or unfortunately there are a number of possible definitions of trophic level. We illustrate two of them here.

The length of a path in a digraph is the number of arcs included in the path. The shortest path between two vertices $x$ and $y$ in a digraph is the path between $x$ and $y$ of shortest length among all such paths. The longest path between two vertices $x$ and $y$ in a digraph is the path between $x$ and $y$ of longest length. We use these definitions of path length to give various definitions of trophic level in a food web. The first is a commonly used definition of trophic level, derived from the trophic level of species in a chain: 


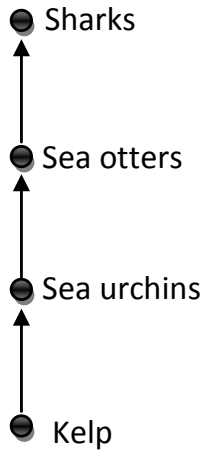

Figure 2: A food chain.

Remark 3. Definition - Option 1 The trophic level of species $X$ is:

i. $\quad 0$, if $X$ is a primary producer in the food web (a species that does not consume any species in the food web)

ii. $\quad k$, if the shortest path from a level 0 species to $X$ is of length $k$.

Example from food web in Figure 1:

\begin{tabular}{|l|l|l|}
\hline Species & Trophic level & Shortest path \\
\hline Kelp & 0 & \\
\hline Sea urchins & 1 & Kelp-Sea urchins \\
\hline Small fishes & 1 & Kelp-Small fishes \\
\hline Large crabs & 2 & Kelp-Sea urchins-Large crabs \\
\hline Sea otters & 2 & Kelp-Sea urchins-Sea otters \\
\hline Sharks & 3 & Kelp-Sea urchins-Sharks \\
\hline
\end{tabular}

Table 2: Trophic level using Option 1 definition of the food web in Figure 1.

Activity 2a. Expand this table to include the trophic level of all species given in Table 1. Answer the following questions before moving on:

1. Do you see any challenges to using the shortest path for computing trophic level?

2. Large crabs are direct prey of sea otters, so would you think they would have different trophic levels?

3. Can you think of an alternative definition of trophic level? 
Remark 4. Definition-Option 2 The trophic level of species $X$ is:

i. $\quad 0$, if $X$ is a primary producer in the food web (a species that does not consume any species in the food web)

ii. $\quad k$, if the longest path from a level 0 species to $X$ is of length $k$.

\begin{tabular}{|l|l|l|}
\hline Species & Trophic level & Longest path \\
\hline Kelp & 0 & Kelp-Sea urchins \\
\hline Sea urchins & 1 & Kelp-Small fishes \\
\hline Small fishes & 1 & Kelp-Sea urchins-Large crabs \\
\hline Large crabs & 2 & Kelp-Small fishes-Sea otters \\
\hline Sea otters & 3 & $\begin{array}{l}\text { Kelp-Small fishes-Large } \\
\text { crabs-Sea otters-Sharks }\end{array}$ \\
\hline Sharks & 4 &
\end{tabular}

Table 3: Trophic level using Option 2 definition of the food web in Figure 1.

Notice that the highest trophic level is now four and that sharks have a higher level using this definition than they did with the other definition, and that large crabs and sea otters now have different trophic levels. Importantly, this definition of trophic level using the longest path satisfies the following assumption:

Remark 5. Assumption If species $X$ is a predator of species $Y$, then the trophic level of species $X$ is greater than the trophic level of species $Y$.

\section{Activity $2 b$.}

1. Complete your table of trophic levels in Activity 2 a to include the trophic level using the longest

2. An ecological rule of thumb is that about $10 \%$ of the energy passes from one trophic level to another. Compare these two definitions of trophic level relative with regard to energy loss. For example, if kelp starts with 1 million units of energy, how much energy is left for sharks using each definition?

Neither of these definitions is entirely satisfactory, since each has its limitations in determining a hierarchical structure of the food web; the first does not satisfy the assumption, and neither one reflects the number of species that are direct or indirect prey of a species. To solve this problem, we combine the length of the longest path and the number of species that are direct or indirect prey and call it the trophic status of a species.

The trophic status of a species $u$ is defined as: 


$$
T(u)=\sum_{k} k n_{k}, \quad \begin{aligned}
& \text { where } n_{k} \text { is the number of species } \\
& \text { whose longest path to } u \text { has length } k .
\end{aligned}
$$

For example, the trophic status of sea otters as shown in Table 4 is computed as follows:

- The longest path to sea otters from kelp is 3 , thus when $k=3, n_{k}=1$.

- The longest path from small fishes to sea otters is 2 , thus when $k=2, n_{k}=1$.

- The longest path from large crabs to sea otters is 1 , and so is the longest path from sea urchins to sea otters 1 . Thus when $k=1, n_{k}=2$.

Therefore, the trophic status of sea otters is

$$
T(\text { sea otters })=3(1)+2(1)+1(2)=7 \text {. }
$$

The trophic status for kelp, sea urchins, small fishes, large crabs, and sharks are shown in Table 4.

\begin{tabular}{|l|l|l|}
\hline Species & $\begin{array}{l}\text { Trophic level } \\
\text { Definition 2 }\end{array}$ & Longest path \\
\hline Kelp & 0 & 0 \\
\hline Sea urchins & 1 & 1 \\
\hline Small fishes & 1 & 1 \\
\hline Large crabs & 2 & $2(1)+1(1)=3$ \\
\hline Sea otters & 3 & $3(1)+2(1)+1(2)=7$ \\
\hline Sharks & 4 & $4(1)+3(1)+2(1)+1(1)=12$ \\
\hline
\end{tabular}

Table 4: Trophic status for species in the food web in Figure 1.

Activity 2c. Find the trophic status of the species in Table 1.

\subsection{Dominant Species}

There are many ways of describing dominant species in a food web. One way is to regard Keystone species (a predator species whose removal causes additional species to disappear - species who effectively control the nature of the community) as always dominant species. 
We can use our model using digraphs to recognize dominant species in a food web by considering what happens when an arc is removed from the digraph representing the food web.

Species $A$ is dominant if the removal of any arc from a species $B$ to $A$ allows $B$ to have uncontrolled growth, and thus become a new "dominant" species. For example, when we remove the arc from sea urchin to sea otter, sea urchins will have uncontrolled growth and they then become a new "dominant" species in the food web.

Activity 3a. Determine the dominant species in the food web corresponding to Table 1 using the definition of arc removal.

Various other definitions of dominant species exist, some of which are:

a. the most numerous species in a food web can be called dominant;

b. the species which occupies the most space can be called dominant;

c. the species with the highest total body mass can be called dominant or

d. the species that contributes the most energy flow can each be called dominant.

One example of a dominance definition, which incorporates both the number of species that are direct or indirect prey and the extent of energy transfer, is based on the trophic status of the species. This definition resembles the definition of status for people in a community or social network.

Remark 6. Trophic Status Dominant Species A species is dominant in a food web if its trophic status is greater than the number of species in the food web above level 0.

\section{Activity $3 b$.}

a. Determine which species are dominant in the food web corresponding to Table 1 using the trophic status definition.

b. Compare your results using trophic status the results you obtain using the definition involving arc removal.

\subsection{Adding Complexity-Weighted Food Webs and Flow-based Trophic Lev- els}

Not all relationships are the same. For example, you interact differently with your family more than with strangers. Or perhaps you love to eat ice cream, and eat as few brussels sprouts as 


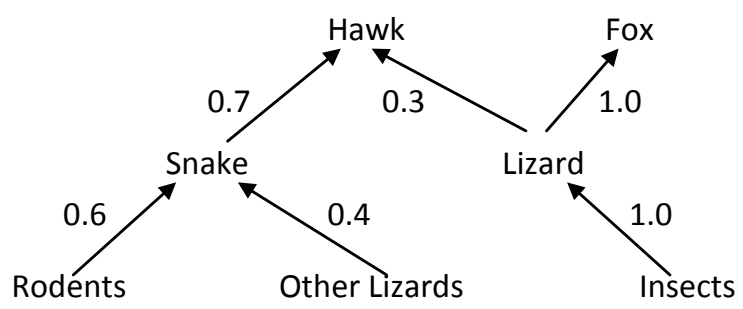

Figure 3:

possible. While both ice cream and brussels sprouts are foods, given an unlimited source of both, would you eat an equal amount of both? Probably not! Similarly, species may eat much more of one species of prey than another. We model this by putting numbers, or weights, on the arcs to indicate food preferences. Consider the food web in Figure 3:

The weight of an arc, called $w_{i j}$, is the proportional food contribution of one vertex $(i)$ to another vertex $(j)$ in the food web. For example, the weight 0.6 on the arc from rodents to snakes reflects that snakes eat "rodents" more than "other lizards" in a ratio of 6 to 4 . Specifically, $60 \%$ of a snake's diet comes from "rodents," while $40 \%$ of its diet comes from "other lizards." The sum of the weights of the ingoing arcs to a species is 1 since the set of ingoing arcs represents the full diet of the species.

Weighting is more important than you might realize. Looking at hawks, it's clear that they eat both snakes and lizards. However, since snakes are weighted so much more heavily, the removal of snakes from this digraph would shift hawk diets dramatically. Instead of eating lizards $30 \%$ of the time, they would eat lizards $100 \%$ of the time. Poor lizards! Lucky insects! When snakes are removed, hawks only eat lizards, which would dramatically decrease the lizard population. This in turn would vastly decreases lizard predation on insects, allowing insects to grow uncontrolled. Now consider what would happen if lizards were initially $90 \%$ of hawk diets (instead of $30 \%$ ). Would the insect population increase so dramatically with the removal of snakes? Probably not! This is important if you are an insect, or anything that eats an insect, or anything that an insect eats. Therefore, it is important to consider the arc weight when predicting indirect changes that trickle down through a food web. In other words, arc weight matters!

Remark 7. Flow-based Trophic Level Weighting the edges of a food web or corresponding digraph gives new alternative definitions of trophic level that take these weights into account. One such definition is called the flow-based trophic level, or TL:

$$
\begin{aligned}
T L(\text { species } i) & =1+\sum(\text { weight of each food source for } i) \times T L(\text { food source }) \\
T L(i) & =1+\sum_{i}\left(w_{i j}\right) \times T L(\text { food source } j)
\end{aligned}
$$


Note that $T L($ primary producer $)=1$. For example, $T L($ snake $)=1+.6(1)+.3(1)=1.9$, whereas $T L($ lizard $)=1+1(1)=2$. Even though under either the shortest path or longest path definitions, the snake and the lizard have the same trophic level, under the flow-based trophic level definition the lizard has a slightly higher flow-based trophic level than the snake.

Activity 4.

a. Finish the calculations for flow-based trophic level for the food web given in Figure 3.

b. What happens to the flow-based trophic level for various species if an arc is removed? Try it by removing the arc from snake to hawk.

\section{Competition Graphs and Habitat Dimension}

We will now use food webs, which model predator-prey relationships, to create additional models to determine the dimension of community habitats from these relationships.

\subsection{Competition Graphs}

Given a food web, create a new (undirected) graph as follows. The vertices are the species in the community and there is an edge between species $a$ and species $b$ if and only if $a$ and $b$ have a common prey, i.e., if there is some $x$ so that there are arcs from $a$ to $x$ and $b$ to $x$ in the food web.

If we take our canonical example from Figure 1 we get a very simple competition graph with one edge and four independent vertices as shown in Figure 4:

Activity 5. Draw the competition graph for the following food web:

Remark 8. Note The competition graph solution for Activity 5 appears at the end of this article.

\subsection{Interval Graphs and Habitat Dimension}

A graph is an interval graph if we can find a set of intervals on the real line so that each vertex is assigned an interval and two vertices are joined by an edge if and only if their corresponding intervals overlap.

Interval graphs have been very important in genetics. They played a crucial role in the physical mapping of DNA and more generally in the mapping of the human genome. 


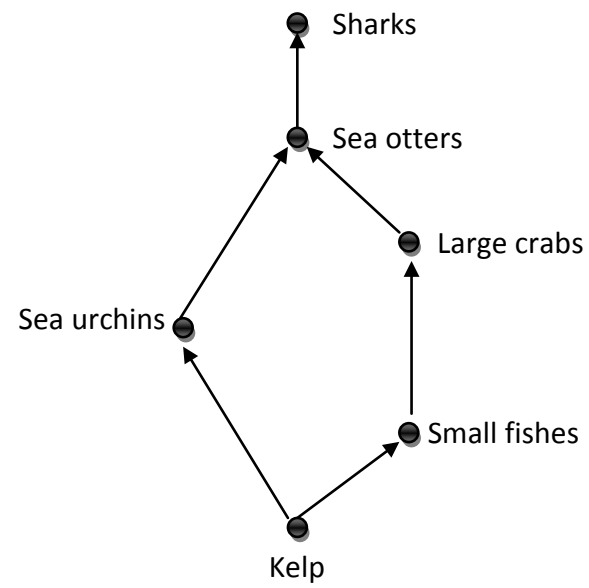

Food Web

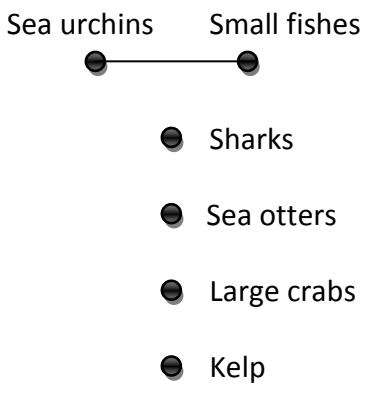

Competition Graph

Figure 4: A food web and its corresponding competition graph.

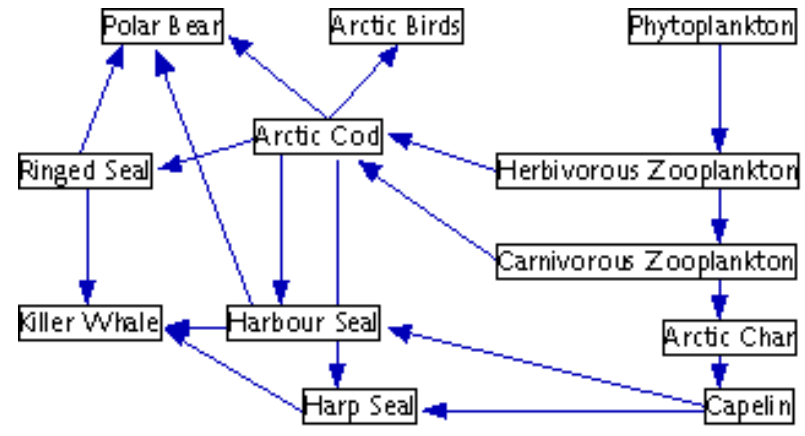

Figure 5: Foodweb from Wikimedia Commons.

Given a competition graph, we want to determine if it is an interval graph.

We need to find intervals on the real line for each vertex so that the intervals corresponding to two vertices overlap if and only if there is an edge between the two vertices.

If $G$ is the competition graph corresponding to a real community food web, and $G$ is an interval graph, then the species in the food web have one-dimensional habitats or niches, and this single dimension applies to each species in the web. This one dimension might be determined by temperature, moisture, $\mathrm{pH}$, or a number of other things.

We have not described the endpoints of the intervals in Figure 6. It would be easy to do so, for example, consider the possible correspondence: 


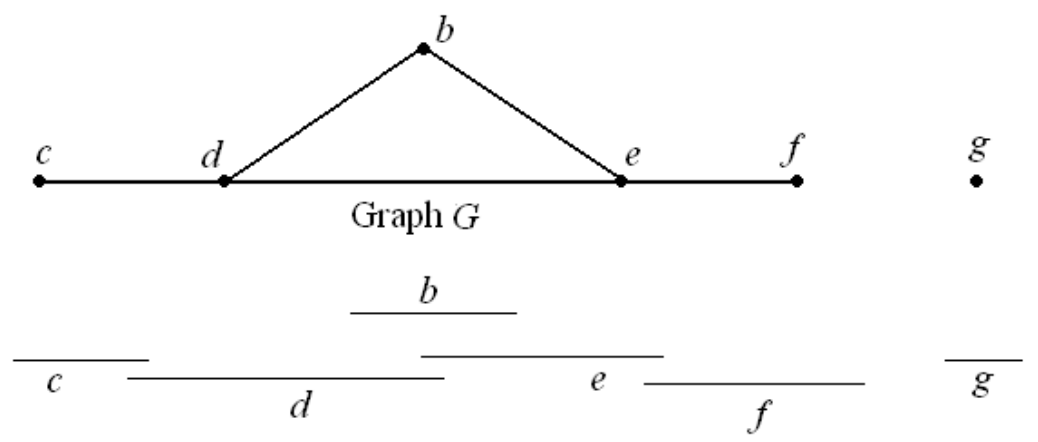

A set of intervals, which correspond to graph $G$, and which overlap if and only if they correspond to an edge in $G$.

Figure 6: $G$ and a demonstration that $G$ is an interval graph.

$\begin{array}{lll}c & \text { to } & {[-2,0]} \\ d & \text { to } & {[-1,2]} \\ b & \text { to } & {[1,4]} \\ e & \text { to } & {[1.5,6]} \\ f & \text { to } & {[5,7]} \\ g & \text { to } & {[9,11]}\end{array}$

Remark 9. Note The size of the interval makes no difference, nor does whether the intervals are open (do not include the endpoints) or are closed (include the endpoints).

If we change the graph slightly to $H$, by adding vertex $a$ and edge $\{a, b\}$, we don't get an interval graph. There is no place to put an interval corresponding to a which overlaps the interval for $b$, but which overlaps the intervals for neither $d$ nor $e$.

Remark 10. Note Independent vertices, like g, do not change the nature of the competition graph in terms of it being an interval graph or not.

\section{Activity 6.}

a. Is the competition graph from Activity 5 an interval graph? Show the interval representation.

b. Give some examples of graphs that are not interval graphs.

c. Can you find a real community food web that has a competition graph that is not an interval graph?

Some graphs, like $H$ shown in Figure 7, cannot be represented by intervals on the real line. Can $H$ be represented by intersecting rectangles in the plane (2-dimensional space)? 


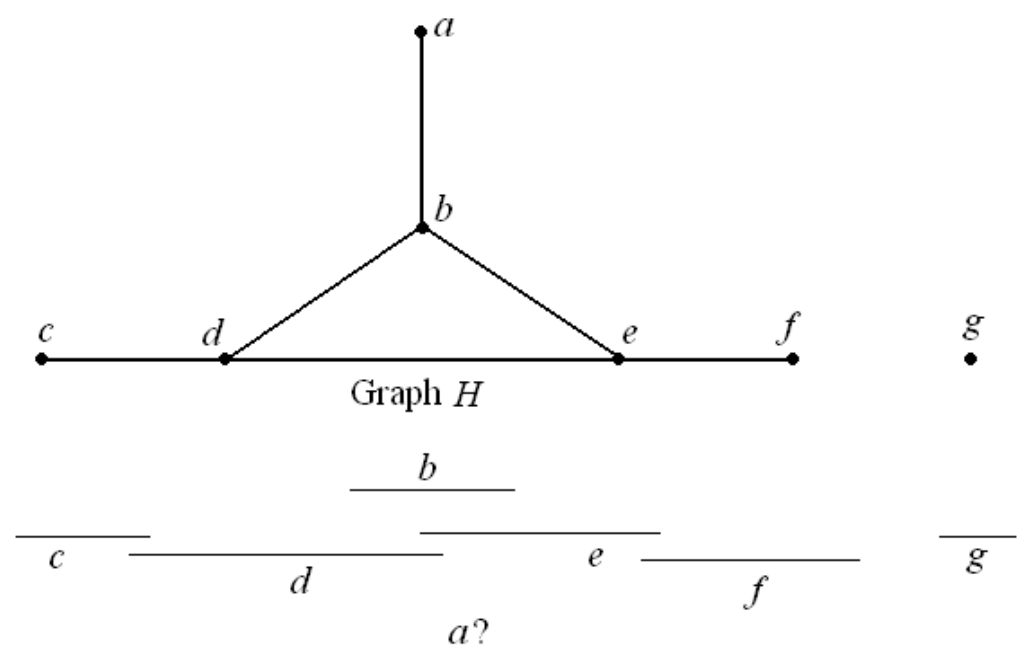

An attempt to find intervals corresponding to graph $H$

Figure 7: Non-interval graph $H$.

More generally, we can consider ways to represent graphs where the edges correspond to intersections of boxes in Euclidean space. An interval on the line is a one-dimensional representation. If we find a representation of a graph where the vertices correspond to rectangles in two-dimensional space so that two rectangles intersect if and only if the corresponding vertices are connected by an edge, then we have a 2-dimensional representation as shown in Figure 8.

Remark 11. Definition The boxicity of $G$ is the smallest $p$ so that we can assign to each vertex of $G$ a box in Euclidean p-space so that two vertices are connected by an edge if and only if their corresponding boxes overlap.

The term boxicity is well-defined [5], but hard to compute [5]. There are fast algorithms to test if a graph is an interval graph, but if it is not an interval graph then there are no fast ways of telling the boxicity of the graph.

The example in Figure 8 shows a graph $G$ of boxicity 2 and overlapping 2-dimensional rectangles to represent $G$.

Different factors determine a species normal healthy environment, factors such as moisture, temperature, and $\mathrm{pH}$. We can use each such factor as a dimension. Then the range of acceptable values on each dimension is an interval. In other words, each species can be represented as a box in Euclidean space, and the box represents its ecological niche.

For example, in Figure 9, the niche of the species might be determined by a temperature between 10 and 15 degrees centigrade, moisture level between 1 and 2, and $\mathrm{pH}$ between 7 and 8 .

Activity 7. Can you find a 2-dimensional box (rectangle) representation for the graph $H$ shown in Figure 7? What does it mean if you can, and what does it mean if you can't? 

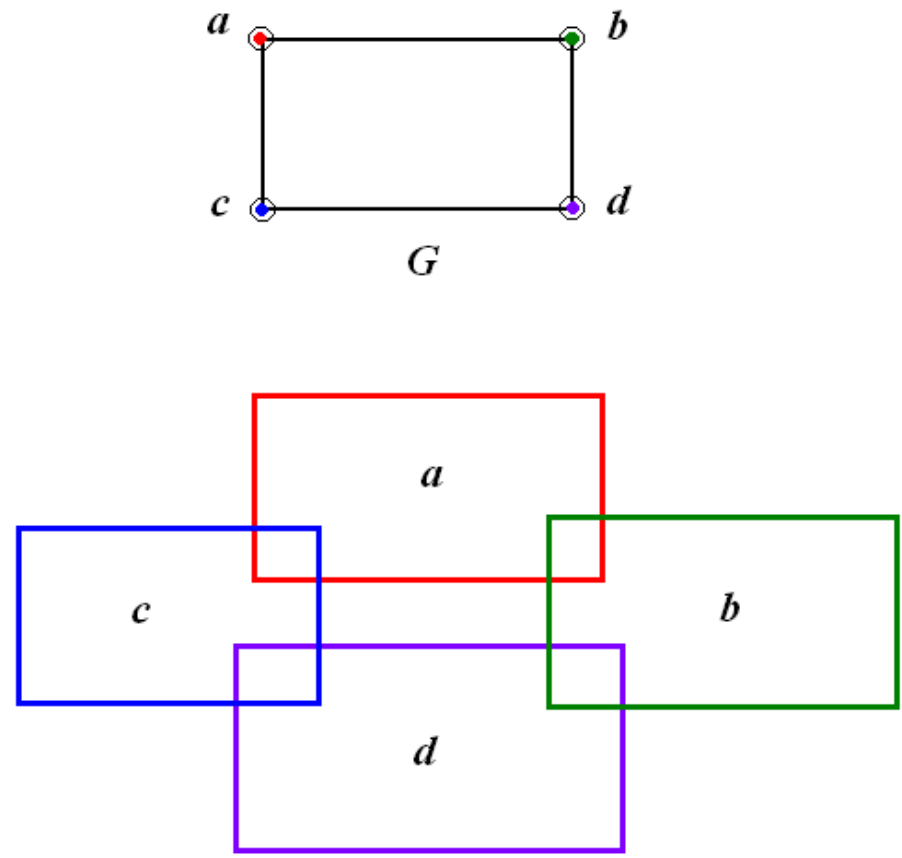

Figure 8: A 2-dimensional box representation of the graph $G$ that is a square.

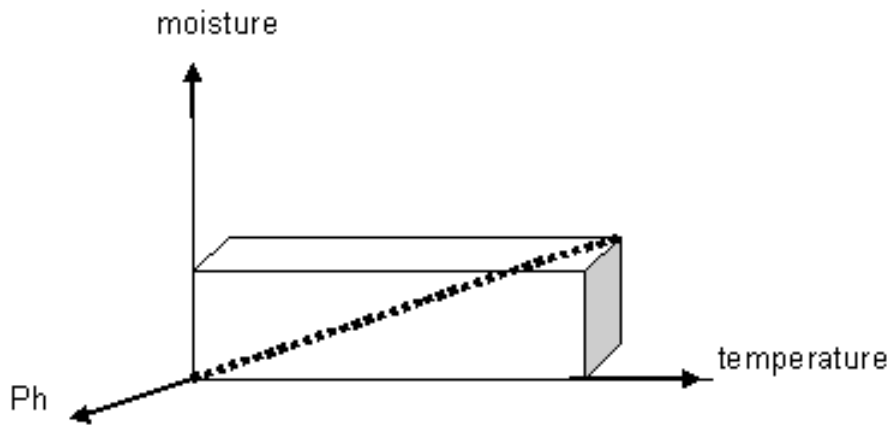

Figure 9: A 3-dimensional species habitat niche represented by temperature, moisture, and $\mathrm{pH}$.

In the 1960's, Joel Cohen found that generally food webs arising from "single habitat ecosystems" (homogeneous ecosystems) have competition graphs that are interval graphs. This remarkable empirical observation of Joel Cohen's [1], that real-world competition graphs are usually interval graphs, has led to a great deal of research on the structure of competition graphs and on the relation between the structure of digraphs and their corresponding competition graphs. It has also led to a great deal of research in ecology to determine just why this might be the case. Is it that there is really only one overriding dimension that controls habitat (niche) formation for a community of species? 
Maybe this should not be surprising statistically. Models for randomly generating food webs have been created and the probability that the corresponding competition graph is an interval graph was calculated. Much of Cohen's Food Webs and Niche Space [1] takes this approach. But Cohen, Komlos, and Mueller [2] showed that under this model, the probability that a competition graph is an interval graph goes to 0 as the number of species increases. In other words it should be highly unlikely that competition graphs corresponding to food webs are interval graphs.

Activity 8. Can you find a competition graph with boxicity 3? In other words find a graph where there is no rectangle representation of the vertices, but there is a 3-dimensional box representation of the vertices so that there is an edge between two vertices if and only if the boxes overlap in 3-dimensional space.

Could one analyze the properties of competition graphs to better understand the underlying food web (directed graph)? In other words, can we characterize the directed graphs whose corresponding competition graphs are interval graphs? This is a fundamental open question in applied graph theory. Indeed there is no forbidden list of digraphs (finite or infinite) such that when these digraphs are excluded, one automatically has a competition graph which is an interval graph. This problem is one which undergraduates with only the knowledge of this paper can tackle.

A second fundamental question, an ecology question, is whether or not it is possible that the habitat or niche of the species in a community food web is truly based on one overriding component, such as temperature or moisture or $\mathrm{pH}$, and if so can one determine what that overriding component is for a specific community food web?

\section{References}

[1] J. E. Cohen. Food webs and niche space. Princeton University Press, Princeton, New Jersey, 1978.

[2] J. E. Cohen, J. Komlos, T. Mueller. The probability of an interval graph and why it matters. Proc. Symposium on Pure Math, 34 (1979), 97-115.

[3] M. B. Cozzens. Integrating mathematics and biology in the high school curriculum. BioMath in the Schools, DIMACS Book Series, American Math Society, 2010.

[4] M. B. Cozzens, N. Crisler, T. Fleetwood. Food webs, COMAP: Lexington MA, 2010.

[5] M. B. Cozzens, F. S. Roberts. Computing the boxicity of a graph by covering its complement by cointerval graphs. Discrete Applied Math, 6 (1983), 217-228.

[6] L. Goldwasser, J. Roughgarden. Construction and analysis of a large Caribbean food web. Ecology, 74 (1993), No. 4., 1216-1233.

[7] A. Hastings, M. A. Palmer. A bright future for biologists and mathematicians. Science, 299 (2003), 2003-2004. 
[8] J. H. Jackson. Bioinformatics and genomics. in Math \& Bio 2010: Linking Undergraduate Disciplines, L.A. Steen (ed.), Mathematical Association of America, 2005, 51-61.

[9] R. W. Morris, C. A. Bean, G. K. Farber, D. Gallahan, A. R. Hight-Walker, Y. Liu, P. M. Lyster, G. C. Y. Peng, F. S. Roberts, M. Twery, J. Whitmarsh. Digital biology: an emerging and promising discipline. Trends in Biotechnology, 23 (2005), 113-117.

[10] R. T. Paine. Food web complexity and species diversity. American Naturalist, 100 (1966), No. 910, 65-75.

[11] F. S. Roberts, Discrete mathematical models with applications to social, biological, and environmental problems. Prentice-Hall, Engelwood Cliffs, NJ, 1976, 111-140.

\section{Websites and Powerpoints:}

http : //www.branson.org/depts/science/marbio/Food Web.html

http : //octopus.gma.org/surfing/human/foodweb.html

http : //two.ucdavis.edu/aking/mam99/graphics/foodweb

Untangling Food Webs to Study Niche Space and Trophic Level Energetics with Graph Theoretic Visualization. John R. Jungck, Don Rohr,Yang Yang, Department of Biology; Tony Abell, Department of Mathematics and Computer Science; Rama Viswanathan, Departments of Chemistry and Mathematics and Computer Science; Beloit College (powerpoint available through J. Jungck).

Food Webs, Competition Graphs, and Biodiversity by M. Cozzens at http : //www.dimacs.rutgers.edu/ midgec

\section{Competition graph solution for Activity 5:}
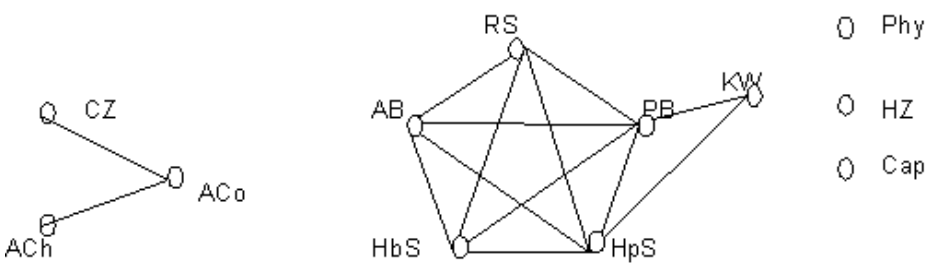